Revue d'histoire de l'Amérique française

सQ REVUE D.HISTOIRE DE L'AMÉRIQUE FRANÇAISE

\title{
Réplique au commentaire du Père Campeau
}

\section{Peter N. Moogk}

Volume 29, numéro 4, mars 1976

URI : https://id.erudit.org/iderudit/303488ar

DOI : https://doi.org/10.7202/303488ar

Aller au sommaire du numéro

Éditeur(s)

Institut d'histoire de l'Amérique française

ISSN

0035-2357 (imprimé)

1492-1383 (numérique)

Découvrir la revue

Citer cette note

Moogk, P. N. (1976). Réplique au commentaire du Père Campeau. Revue d'histoire de l'Amérique française, 29(4), 571-576.

https://doi.org/10.7202/303488ar d'utilisation que vous pouvez consulter en ligne.

https://apropos.erudit.org/fr/usagers/politique-dutilisation/ 


\title{
RÉPLIQUE AU COMMENTAIRE DU PÈRE CAMPEAU
}

\author{
Peter N. MOOGK \\ Forteresse de Louisbourg
}

Quand un chercheur aussi remarquable que le père Lucien Campeau se porte à la défense de la version traditionnelle de l'histoire de l'École des arts et métiers de Saint-Joachim, ses propos méritent qu'on y prête attention. Pour ma part, je regrette qu'il ait trouvé mon texte confus, car si tel est le cas la responsabilité en incombe davantage à l'auteur qu'au traducteur qui a fait un travail fort convenable. Quoi qu'il en soit, il demeure que le père Campeau a mal compris des portions de mon article.

Il n'était pas facile de voir clair dans le mélange de faits, de souhaits qui se veulent des réalités, d'interprétations fantaisistes, auquel avait abouti l'étude des documents concernant le Petit Séminaire de Québec, son annexe du cap Tourmente et l'école élémentaire située au même endroit. J'aurais souhaité affirmer après d'autres qu'une autre école existait en effet, une école des arts et métiers sise au cap Tourmente en la paroisse de Saint-Joachim. J'avais commencé mes recherches dans l'espoir d'en vérifier l'existence. Malheureusement, un examen attentif des données oblige avec regret à conclure que cette école n'a jamais existé. L'origine de la fable remonte à certains ouvrages du $\mathrm{XIX}^{\mathrm{e}}$ siècle dont les auteurs tentèrent de décrire le système d'éducation utilisé pour les garçons qui vivaient dans les fermes du Séminaire au cap Tourmente, en se servant d'analogies qui leur étaient contemporaines. Dans des ouvrages subséquents, ces analogies douteuses donnèrent lieu à l'affirmation selon laquelle une école des arts et métiers, comme celles qu'on trouvait au Québec au début du $\mathrm{XX}^{\mathrm{e}}$ siècle, existait déjà au cap Tourmente à l'époque de Mgr de Laval. Du reste les biographes de celui-ci sont en partie responsables des avatars de cette fable, désireux qu'ils étaient de donner au premier évêque de la Nouvelle-France crédit d'avoir mis sur pied une école technique. Convaincu de l'existence de cette école, Amédée Gosselin tenta en 1911 d'en fournir une preuve documentaire qui reste de nature ambiguë et qui s'applique vraisemblablement aux trois institutions mentionnées plus haut. Pris un à un, les documents auxquels il fait référence ne sont pas probants. Plusieurs détails mentionnés par Gosselin et embellis par ceux qui ont écrit après lui sont les fruits de l'imagination et non les résultats d'une recherche sans passion. On en arrive ainsi à la conclusion que non 
seulement l'existence de l'École des arts et métiers est improbable, mais qu'à la lumière de ce que nous savons sur l'apprentissage des métiers en Nouvelle-France, toute cette histoire véhiculée par la tradition est un pur anachronisme. Voilà donc le triste verdict que contient mon article.

Contrairement à ce à quoi je pouvais m'attendre, le père Campeau n'a présenté aucune preuve nouvelle ou incontestable en faveur de l'existence de l'École des arts et métiers. Il est quand même surprenant qu'un historien à la fois aussi prudent et aussi accompli défende dans son intégrité cette histoire d'école avec toutes ses contradictions et ses distorsions. Puisqu'il ne s'en tient lui-même dans sa critique qu'à quatre documents, peut-être estime-t-il que l'ensemble de la preuve au sujet de la prétendue fondation est insoutenable. Même dans ces conditions, si on se limite au sens de ces documents sans y injecter nos propres pensées, on constate qu'ils ne justifient d'aucune façon la légende de l'École des arts et métiers du Cap Tourmente.

Que faut-il penser de cette phrase de Denonville datée de novembre 1685 et dont je souligne moi-même certains mots: "J'ay trouvé icy dans le seminaire de l'Evesché le commencement de deux establissements», c'est-à-dire "deux maisons ou l'on retire des Enfans po [ur] les instruire»? Le père Campeau a sans doute raison d'identifier l'une de ces «maisons» comme étant le Petit Séminaire de Québec. S'ensuit-il toutefois que l'autre «maison» soit l'École des arts et métiers de Saint-Joachim? Pour arriver à cette interprétation, le père Campeau change les mots de Denonville et lui fait dire qu'il a trouvé deux établissements «déjà formés» et chapeautés par le Séminaire de Québec. C'est une modification importante de l'affirmation du gouverneur. Denonville n'est plus en mesure de nous expliquer ce qu'il entendait par "le commencement de deux establissements» et ce n'est certes pas lui rendre justice que de lui imposer nos propres pensées. Quand il parle de la seconde "maison» en disant qu' «on y met ceux qui ne sont propres q[ue] po[u]r estre artisans, et a ceux la on apprend des mestiers », peut-être ne devrionsnous comprendre que ce précisément qui est écrit, c'est-à-dire une résidence pour les garçons qui apprennent des métiers. Denonville n'affirme pas que l'apprentissage se fait dans cette «maison». On sait que des jeunes garçons du Petit Séminaire, peu doués pour les études, étaient confiés, comme apprentis, aux engagés de l'institution à Québec et il serait fort plausible qu'ils aient vécu séparés de leurs condisciples qui suivaient le programme régulier des études. 
Si on appelle cette «maison» une école des arts et métiers, on serait fondé de nommer "manufacture» une simple maison de pension pour ouvriers.

Il y a plus. En dépit de l'affirmation de Denonville selon laquelle cette "maison» se trouvait "dans le séminaire» à Québec, il nous faudrait comprendre que, comme la santa casa de Loretto, elle se serait envolée au Cap Tourmente. Par un habile argument $a d$ hominem, les faits consignés à la page 10 de mon article me sont présentés comme une preuve, devant laquelle je suis resté aveugle, de l'existence d'une École de métiers au Cap Tourmente. La dite preuve viendrait du recensement de 1681 que je mentionne dans mon article en rapport avec l'institution mise en cause. Comme je l'ai dit, le recensement mentionne la présence dans les fermes du Séminaire de la seigneurie de Beaupré, de sept garçons, d'un couple âgé, de deux serviteurs et de Claude Denis. On peut voir là une preuve de l'existence d'une petite école à cet endroit avant 1685, et j'ai reconnu cette possibilité à la page 10 de mon article. À la façon d'Amédée Gosselin, le père Campeau identifie cette école élémentaire dont l'existence est bien connue par la suite, à une école des arts et métiers. Il ignore le fait que le même recensement mentionne la présence à Québec et non au Cap Tourmente, de deux des garçons dont nous savons qu'ils firent leur apprentissage avec les artisans du Séminaire. On peut par ailleurs croire que le couple âgé et les deux serviteurs étaient occupés plus qu'il n'en faut avec leurs soixantedix têtes de bétail et leurs cent arpents de terre à cultiver. J'ai déjà parlé dans mon article de l'hypothèse selon laquelle le père Louis Soumande, qui était dans la paroisse, y aurait assumé les fonctions d'enseignant. Je n'y reviens pas. Ce qui ne nous laisse plus qu'un jeune homme éduqué dans les humanités, qui, si l'on en croit notre critique, aurait formé sept jeunes garçons à des métiers variés en plus de leur apprendre à lire et à écrire.

Comment peut-on voir dans ce petit groupe de garçons, sous la possible tutelle d'un instituteur, une école des arts et métiers en plein essor? De tous les garçons dont les Annales du Séminaire nous disent qu'ils ont été envoyés au Cap Tourmente, nous n'en connaissons qu'un seul qui a fait l'apprentissage d'un métier. Quant aux autres qui devinrent artisans, aucune mention ne les rattache au Cap Tourmente. Ce garçon s'appelle Jean-Baptiste Lamusette. Il entra au Petit Séminaire en 1678, fut envoyé au Cap Tourmente qu'il quitta «ayant appris le métier de masson». On sait qu'il y avait des maçons dans la seigneurie de Beaupré et que près de Beauport 
des carrières donnaient du travail à plusieurs tailleurs de pierre. J'ai émis l'hypothèse que Lamusette a pu apprendre son métier de l'un de ces maçons. Cette hypothèse serait à rejeter car, selon le père Campeau, il était de règle au Séminaire que les élèves et les employés dépendent exclusivement de l'institution et l'apprentissage sous la surveillance d'un artisan indépendant aurait été contraire à cette règle. Il semble bien, en effet, qu'à l'origine une telle politique existait. Mais en ce qui concerne les employés, elle avait déjà été abandonnée à la date qui nous intéresse, le Séminaire ayant été à plusieurs reprises déçu de la conduite des engagés venus de France avec un contrat de trois ans. Les livres de compte du Séminaire montrent qu'après 1675 , l'institution employait, au mois, à la journée ou à contrat fixe, des ouvriers canadiens travaillant pour leur compte. En 1682, le procureur du Séminaire, malgré le coût, en recommande l'engagement «dautant quon est souvent trompé en ceux quon fait passer de france». ${ }^{1}$ Aussi n'est-il pas impossible que Jean-Baptiste Lamusette ait appris son métier d'un maçon indépendant, employé dans les fermes du Séminaire. De fait, on sait que les maçons étaient fort désireux d'avoir des apprentis qui les aidaient dans leur travail.

On nous donne enfin comme preuves, l'affirmation de SaintVallier à l'hiver de 1685-1686 et celle contenue dans l'acte de donation de Laval en 1693, voulant que les enfants du Cap Tourmente soient élevés dans l'apprentissage, entre autres choses, "des mestiers». Lorsque, comme en 1705 , est décrite la tâche de ces "enfants de travail», on voit qu'elle consiste à apprendre le travail de la terre et non pas celui d'un métier spécialisé. Toutefois, comme je l'ai dit aux pages 17 et 18 de mon article, je pense qu'il est possible que quelques garçons aient appris un métier au Cap Tourmente; ce qui n'entraîne absolument pas l'existence d'une école des arts et métiers. Avant de conclure que l'engagement dans «des métiers» signifie nécessairement la pratique de métiers spécifiques, il faut se rappeler que le mot métier avait alors une acception beaucoup plus large qu'aujourd'hui. Le Dictionnaire universel de Furetière (1690) donne du mot sept significations différentes. Sauf "gens de métiers" qui renvoie nécessairement à artisans, le mot métier pouvait se dire pour profession, travail, devoir, habitude et nécessité. Aussi, dire que les garçons du Cap Tourmente seraient employés dans «des mestiers» signifie probablement qu'ils allaient

1 Archives du Séminaire de Québec, lettre N, no 61:10; cité par Noël Baillargeon, Le Séminaire de Québec sous l'épiscopat de Mgr de Laval (Québec, 1972), 130. 
faire un travail physique, en particulier dans l'agriculture, et parfois dans l'apprentissage d'un métier reconnu lorsqu'un bon artisan se trouvait disponible.

Le père Campeau a apporté une contribution fort valable à l'explication du conflit qui oppose Laval et Saint-Vallier au sujet du Petit Séminaire du Cap Tourmente. Mais elle est en dehors de la question, à moins qu'elle ait eu pour but de montrer que Laval avait bel et bien fondé une école des arts et métiers au même endroit. À part l'acte de donation de 1693, aucun document n'indique que Laval ait fondé quelque école que ce soit au Cap Tourmente. L'argument selon lequel ce qui a pu être une petite école dirigée par Claude Denis était en fait une école des arts et métiers fondée par Laval ne repose sur aucune preuve. Il n'existe pas une seule référence explicite à pareille institution et on ne saurait nommer avec certitude aucun artisan du Séminaire qui ait enseigné à cet endroit.

Il est certain que nous entendrons encore parler de l'École des arts et métiers de Laval à Saint-Joachim. Une lecture imaginative des documents pourra maintenir vivante la légende, et pour plusieurs cela est suffisant. Si on raisonne en disant que, sans preuves contraires, tout est possible, alors l'institution en cause reste une possibilité. Bien sûr, en l'absence de preuves concluantes, on peut parler de ce qui est probable. Mais l'argument de probabilité doit être dans les limites du raisonnable. Il doit posséder une logique interne et s'ajuster étroitement au sens littéral des textes, sans trop d'interpolations. Comme nous l'avons vu pour la lettre de Denonville, le sens des mots doit être modifié pour arriver à prouver que l'école existe. Comme je l'ai dit dans mon article, l'histoire traditionnelle de l'école est remplie de contradictions, d'erreurs et d'inventions. Ce n'est pas là une façon acceptable de faire l'histoire. Ayant examiné les documents, je ne crois pas que l'existence de l'École des arts et métiers soit même une probabilité. Au mieux, ce que l'on peut dire, c'est que quelques garçons placés par le Séminaire au Cap Tourmente y ont appris des métiers comme d'autres garçons du Petit Séminaire, peu doués pour les études, devinrent apprentis à Québec, ou comme de «jeunes clercs» s'initièrent à des métiers dans leur temps libre. À cette époque, pareil apprentissage se faisait habituellement en dehors des cadres d'une institution scolaire.

On m'a reproché de ne pas voir dans les documents ce qu'y ont vu d'autres historiens plus expérimentés. Je sais que je suis exaspérant et que je dois paraître dépourvu d'imagination à force 
d'insister sur le sens littéral des documents. Par ailleurs, peut-être ne vois-je pas dans les manuscrits les références à l'École des arts et métiers du Cap Tourmente tout simplement parce qu'elles n'y sont pas.

Traduction de JEAN BLAIN Université de Montréal 